\title{
SCALING OF POISSON SPHERES AND COMPACT LIE GROUPS*
}

\author{
ALBERT JEU-LIANG SHEU ${ }^{\dagger}$
}

\begin{abstract}
For $n>2$, we show that on the standard Poisson homogeneous space $\mathbb{S}^{2 n-1}$ (including $S U(2) \approx \mathbb{S}^{3}$ ), there exists a Poisson scaling $\phi_{\lambda}$ at any scale $\lambda>0$ that is smooth on each symplectic leaf and continuous globally. A generalization to the case of the standard Bruhat-Poisson compact simple Lie groups endowed with a stronger topology is also valid.
\end{abstract}

Key words. Poisson Lie groups, Bruhat-Poisson structure, covariant Poisson structure, homogeneous Poisson structure, scaling, deformation quantization, compact simple Lie groups.

AMS subject classifications. Primary: 53D17; Secondary: 17B37, 53D55.

Introduction. In connection with the modular automorphism groups [W3], Weinstein showed [W2] that there is no nontrivial smooth scaling $(\phi, \lambda)$, called dilation, of the standard Bruhat-Poisson structure $\pi$ on $S U$ (2) (or the reduced Poisson structure on its homogeneous space $\mathbb{S}^{2}$ ), i.e. a diffeomorphism $\phi$ of $S U(2)$ and a scalar $\lambda \neq 0$ such that $\phi^{*} \pi=\lambda^{-1} \pi$, other than $(\phi, \lambda)=(\iota,-1)$ where $\iota(u):=u^{-1}$ is the inverse map on $S U(2)$. This result is then generalized to all compact groups with Bruhat-Poisson structure by J.-H. Lu [W2].

However a very important geometric structure of a Poisson manifold is its decomposition into (maximal) symplectic leaves [W1] of various dimensions in general, which form some kind of "singular" foliation. Even though such a symplectic foliation has a nice local Poisson product structure [W1], it is not a standard regular foliation with a clean smooth structure everywhere. Instead, the closure of a symplectic leaf may meet many symplectic leaves of lower dimensions, and there further degeneracies of the Poisson structure occur, rendering a weaker sense of smoothness. From this viewpoint, the global smoothness of a scaling or dilation seems too strong a requirement in general. In this paper, we show that if a scaling $\phi_{\lambda} \equiv(\phi, \lambda)$ is required to be only continuous on the whole manifold but smooth on each symplectic leaf of a Poisson manifold, then it exists for all $\lambda>0$ on the Poisson homogeneous space $\mathbb{S}^{2 n-1}$ of the Bruhat-Poisson $S U(n)$. A Liouville vector field generating $\phi_{\lambda}$ is explicitly computed for $S U(2)$. Furthermore, if a standard Bruhat-Poisson compact simple Lie group $K$ is endowed with some stronger topology that is still compatible with the original differential structure on each symplectic leaf of $K$, then a leafwise smooth and globally continuous scaling $\phi_{\lambda}$ exists on $K$ for all $\lambda>0$.

In [Sh1], it is shown that the standard Bruhat-Poisson $S U(2)$ can be quantized by Weyl calculus along all of its symplectic leaves to construct a $\mathrm{C}^{*}$-algebraic deformation quantization of the Poisson structure and yield the $\mathrm{C}^{*}$-algebra $C\left(S U(2)_{q}\right)$ of quantum $S U(2)$. The construction essentially composes a standard Weyl quantization of $\mathbb{C}$ with $\phi_{\lambda}^{*}$ for a family of continuous scalings $\phi_{\lambda}$ of the Poisson $S U$ (2). This method of quantization is intrinsically of "leaf-preserving" type as opposed to the "group-preserving" type, and there is a no-go theorem saying that these two types of quantization are disjoint [Sh2, Sh3]. For general Bruhat-Poisson compact simple Lie groups $K$, faithful leaf-preserving deformation quantizations have not been

\footnotetext{
*Received February 23, 2011; accepted for publication February 2, 2012.

$\dagger$ Department of Mathematics, University of Kansas, Lawrence, KS 66045, USA (sheu@math. ku.edu). Partially supported by University of Kansas, and by the National Center for Theoretical Sciences.
} 
constructed, while group-preserving ones have been found [N] for $S U(n)$. Whether composing a standard Weyl quantization of $\mathbb{C}^{n}$ with $\phi_{\lambda}^{*}$ for a family of continuous scalings $\phi_{\lambda}$ of the Poisson $S U(n)$ results in a deformation quantization that produces the algebra of quantum $S U(n)$ remains to be studied.

The author would like to thank the National Center for Theoretical Sciences and the National Taiwan University for their warm hospitality and the excellent research environment provided during his visit in Fall 2010.

1. Scaling of Poisson $S U(2)$. In this section, we study how the standard Bruhat-Poisson structure on $S U(2)$ can be scaled smoothly leafwise and continuously globally.

We call a family of homeomorphisms $\phi_{t}: M \rightarrow M, t>0$, on a Poisson manifold $(M, \pi)$ a scaling of the Poisson structure of $M$ if $\phi_{t}$ is a diffeomorphism from each symplectic leaf of $M$ onto itself with

$$
\left(\left(\phi_{t}\right)_{*} \pi\right)(x):=\left(D \phi_{t}\right)_{\phi_{-t}(x)}\left(\pi\left(\phi_{-t}(x)\right)\right)=t \pi(x)
$$

and $\phi_{1}(x)=x$ for each $x \in M$. A basic example is the scaling of the standard symplectic structure $\frac{\partial}{\partial x} \wedge \frac{\partial}{\partial y}$ on $\mathbb{C}$ given by the family of diffeomorphisms $\mu_{t}$ of $\mathbb{C}$ defined by

$$
\mu_{t}(w):=\sqrt{t} w
$$

for $t>0$ and $w \in \mathbb{C}$.

Recall that a smooth vector field $X$ (assumed to be complete in this paper for simplicity) is called a Liouville vector field if $[X, \pi]=-\pi$, which if exists, generates a smooth scaling $\phi_{t}:=\alpha_{-X}(\ln t)$ of $\pi$, where $\alpha_{-X}(s)$ with $s \in \mathbb{R}$ denotes the integral flow generated by the vector field $-X$. For example, the smooth vector field

$$
X: w \mapsto \frac{-1}{2} w
$$

whose opposite $-X$ generates $\phi_{t} \equiv \alpha_{-X}(\ln t)=\mu_{t}$ is a Liouville vector field on the standard symplectic manifold $\mathbb{C}$. Generalizing this notion to fit our consideration of non-smooth scalings, we call a continuous vector field $X$ on the Poisson manifold $(M, \pi)$ a Liouville vector field if $\left.X\right|_{L} \in \Gamma(T L)$ is a smooth (tangential) vector field on $L$ and $\left[\left.X\right|_{L},\left.\pi\right|_{L}\right]=-\left.\pi\right|_{L}$ is valid for each symplectic leaf $L$ of $M$.

By embedding $S U(2)$ into $M_{2 \times 2}(\mathbb{C}) \cong \mathbb{C}^{4}$ in the canonical way, we can concretely identify the tangent space $T_{u} S U(2)$ of $S U(2)$ at any $u \in S U(2)$ with the left (multiplicative) translation

$$
L_{u} \mathfrak{s u}(2) \equiv u \mathfrak{s u}(2) \subset M_{2 \times 2}(\mathbb{C})
$$

of the Lie algebra $\mathfrak{s u}(2) \equiv T_{e} S U(2) \subset M_{2 \times 2}(\mathbb{C})$ by the matrix $u$, where $e \equiv I_{2}$ is the unit element of $S U(2)$.

Recall that the standard multiplicative Bruhat-Poisson structure on $S U$ (2) [D1, LW1, VSo1] is generated by

$$
r=\left(\begin{array}{cc}
0 & 1 \\
-1 & 0
\end{array}\right) \wedge\left(\begin{array}{cc}
0 & i \\
i & 0
\end{array}\right) \in \wedge^{2} T_{e} S U(2) \equiv \wedge^{2} \mathfrak{s u}(2)
$$

More precisely, the Poisson 2-tensor $\pi$ of the Bruhat-Poisson $S U$ (2) at

$$
u=\left(\begin{array}{ll}
a & b \\
c & d
\end{array}\right) \in S U(2),
$$


with $a, b, c, d \in \mathbb{C}$ such that $\bar{a}=d, b=-\bar{c}$, and $|a|^{2}+|c|^{2}=1$, is given by

$$
\begin{aligned}
\pi_{u}= & L_{u}(r)-R_{u}(r)=\left(\begin{array}{ll}
a & b \\
c & d
\end{array}\right)\left(\begin{array}{cc}
0 & 1 \\
-1 & 0
\end{array}\right) \wedge\left(\begin{array}{ll}
a & b \\
c & d
\end{array}\right)\left(\begin{array}{ll}
0 & i \\
i & 0
\end{array}\right) \\
& -\left(\begin{array}{cc}
0 & 1 \\
-1 & 0
\end{array}\right)\left(\begin{array}{ll}
a & b \\
c & d
\end{array}\right) \wedge\left(\begin{array}{cc}
0 & i \\
i & 0
\end{array}\right)\left(\begin{array}{ll}
a & b \\
c & d
\end{array}\right) \\
= & \left(\begin{array}{ll}
-b & a \\
-d & c
\end{array}\right) \wedge\left(\begin{array}{cc}
i b & i a \\
i d & i c
\end{array}\right)-\left(\begin{array}{cc}
c & d \\
-a & -b
\end{array}\right) \wedge\left(\begin{array}{ll}
i c & i d \\
i a & i b
\end{array}\right) .
\end{aligned}
$$

In the following, we denote by

$$
L_{0}=\left\{\left(\begin{array}{cc}
a & -c \\
c & \bar{a}
\end{array}\right) \in S U(2): c=\sqrt{1-|a|^{2}} \text { and }|a|<1\right\}
$$

the basic symplectic leaf that plays a crucially important role in the study of BruhatPoisson $S U(2)$ [VSo1]. We also use the notation

$$
P=P_{n}: u \in S U(n) \mapsto u\left(e_{1}\right) \in \mathbb{S}^{2 n-1}
$$

for the fibration projection map, which is a diffeomorphism when $n=2$, where $\left\{e_{i}\right\}_{i=1}^{n}$ is the standard basis of $\mathbb{C}^{n}$.

Proposition. There exists a (continuous and leafwise smooth) scaling $\phi_{t}, t>0$, of the standard Bruhat-Poisson structure of $S U(2)$, which is generated leafwise as $\alpha_{-X}(\ln t)$ by the opposite $-X$ of the (continuous and leafwise smooth) Liouville vector field

$$
\begin{aligned}
X: u & \equiv\left(\begin{array}{cc}
r e^{i \theta} & -\sqrt{1-r^{2}} e^{-i \eta} \\
\sqrt{1-r^{2}} e^{i \eta} & r e^{-i \theta}
\end{array}\right) \in S U(2) \\
& \mapsto\left(\begin{array}{cc}
\frac{\left(1-r^{2}\right) \ln \left(1-r^{2}\right)}{2 r} e^{i \theta} & \frac{\sqrt{1-r^{2}} \ln \left(1-r^{2}\right)}{2} e^{-i \eta} \\
\frac{-\sqrt{1-r^{2}} \ln \left(1-r^{2}\right)}{2} e^{i \eta} & \frac{\left(1-r^{2}\right) \ln \left(1-r^{2}\right)}{2 r} e^{-i \theta}
\end{array}\right) \in T_{u} S U(2) \subset M_{2 \times 2}(\mathbb{C}) .
\end{aligned}
$$

on $(S U(2), \pi)$.

Proof. For $u \in \overline{L_{0}} \subset S U(2)$ with $a=x+i y$ (and $c \geq 0$ ), we have

$$
\begin{aligned}
P_{*}\left(\pi_{u}\right) & =\left(\begin{array}{c}
c \\
-\bar{a}
\end{array}\right) \wedge\left(\begin{array}{c}
-i c \\
i \bar{a}
\end{array}\right)-\left(\begin{array}{c}
c \\
-a
\end{array}\right) \wedge\left(\begin{array}{c}
i c \\
i a
\end{array}\right) \\
& =-2\left(\begin{array}{c}
c \\
-x
\end{array}\right) \wedge\left(\begin{array}{c}
i c \\
-y
\end{array}\right)=-2\left(\begin{array}{c}
c \\
-\operatorname{Re}(a)
\end{array}\right) \wedge\left(\begin{array}{c}
i c \\
-\operatorname{Im}(a)
\end{array}\right) .
\end{aligned}
$$

It is easy to see that $P_{*}\left(\pi_{u}\right)=0$ (and hence $\left.\pi_{u}=0\right)$ at $u=\operatorname{diag}\left(e^{i \theta}, e^{-i \theta}\right) \in U(1) \subset$ $S U$ (2), and hence the subgroup $U(1)$ consists of 0 -dimensional symplectic leaves of $S U(2)$. So the canonical (left) $U(1)$-action on $S U(2)$ keeps the Poisson structure invariant [LW1, VSo1].

Under the diffeomorphism $P$, the element $\operatorname{diag}\left(e^{i \theta}, e^{-i \theta}\right) \in U(1)$ acts on $\mathbb{S}^{3} \approx$ $S U(2)$ as $(a, c) \mapsto\left(e^{i \theta} a, e^{-i \theta} c\right)$. So we get, for

$$
u=\left(\begin{array}{cc}
a & -\bar{c} \\
c & \bar{a}
\end{array}\right) \in S U
$$


in general,

$$
P_{*}\left(\pi_{u}\right)=-2\left(\begin{array}{c}
\bar{c} \\
-\operatorname{Re}\left(\frac{a c}{|c|}\right) \frac{c}{|c|}
\end{array}\right) \wedge\left(\begin{array}{c}
i \bar{c} \\
-\operatorname{Im}\left(\frac{a c}{|c|}\right) \frac{c}{|c|}
\end{array}\right)
$$

which vanishes at $u \in U(1)$ (where we formally take $c /|c|:=1$ if $c=0$ ).

First we perform a change of variables by the diffeomorphism

$$
\psi: u \in L_{0} \mapsto z=\frac{a}{c}=\frac{a}{\sqrt{1-|a|^{2}}} \in \mathbb{C},
$$

and get

$$
\psi_{*}\left(\pi_{u}\right)=\frac{-2}{|c|^{2}}(1 \wedge i)=-2\left(1+|z|^{2}\right)(1 \wedge i) \in \wedge^{2} T_{z} \mathbb{C} .
$$

Under another change of variables by the diffeomorphism

$$
\tau: z=r e^{i \theta} \in \mathbb{C} \mapsto w=\sqrt{2^{-1} \ln \left(1+r^{2}\right)} e^{-i \theta} \in \mathbb{C},
$$

we get the standard symplectic 2 -tensor $\frac{\partial}{\partial x} \wedge \frac{\partial}{\partial y}$ on $\mathbb{C}$, i.e.

$$
\begin{aligned}
& \tau_{*}\left(\psi_{*}\left(\pi_{u}\right)\right)=\tau_{*}\left(-2\left(1+r^{2}\right)\left(\frac{\partial}{\partial x} \wedge \frac{\partial}{\partial y}\right)_{z}\right)=-2 \tau_{*}\left(\left(1+r^{2}\right)\left(\frac{1}{r} \frac{\partial}{\partial r} \wedge \frac{\partial}{\partial \theta}\right)_{z}\right) \\
& =-2\left(\frac{1+r^{2}}{r}\right)\left(\frac{1}{2} \frac{r}{\left(1+r^{2}\right) \sqrt{2^{-1} \ln \left(1+r^{2}\right)}} \frac{\partial}{\partial r} \wedge\left(-\frac{\partial}{\partial \theta}\right)\right)_{w} \\
& =\left(\frac{1}{\sqrt{2^{-1} \ln \left(1+r^{2}\right)}} \frac{\partial}{\partial r} \wedge \frac{\partial}{\partial \theta}\right)_{w}=\left(\frac{\partial}{\partial x} \wedge \frac{\partial}{\partial y}\right)_{w} \in \wedge^{2} T_{w} \mathbb{C} \text {. }
\end{aligned}
$$

Under the transformation $\tau$, the canonical smooth scaling $\mu_{t}: w \mapsto \sqrt{t} w, t>0$, of the standard symplectic structure $\frac{\partial}{\partial x} \wedge \frac{\partial}{\partial y}$ on $\mathbb{C}$ is equivalent to a scaling

$$
z=r e^{i \theta} \in \mathbb{C} \mapsto \sqrt{\left(1+r^{2}\right)^{t}-1} e^{i \theta} \in \mathbb{C}
$$

of the Poisson structure $\psi_{*} \pi$ on $\mathbb{C}$, which in turn, gives rise to a scaling of $\pi$ on $L_{0}$ defined by

$$
\phi_{t}:\left(\begin{array}{cc}
r e^{i \theta} & -\sqrt{1-r^{2}} \\
\sqrt{1-r^{2}} & r e^{-i \theta}
\end{array}\right) \in L_{0} \mapsto\left(\begin{array}{cc}
\sqrt{1-\left(1-r^{2}\right)^{t}} e^{i \theta} & -\sqrt{\left(1-r^{2}\right)^{t}} \\
\sqrt{\left(1-r^{2}\right)^{t}} & \sqrt{1-\left(1-r^{2}\right)^{t}} e^{-i \theta}
\end{array}\right) \in L_{0} .
$$

Note that this formula for $\phi_{t}$ can also be continuously applied to $u \in U(1)=\partial\left(L_{0}\right)$ with $r=1$ and yield $\phi_{t}(u)=u$ for $u \in U(1)$. Also note that by our construction, the map

$$
a=r e^{i \theta} \in \overline{\mathbb{D}} \mapsto a_{t}=\sqrt{1-\left(1-r^{2}\right)^{t}} e^{i \theta} \in \overline{\mathbb{D}}
$$

is smooth on the open unit disk $\mathbb{D}$ and continuous on $\overline{\mathbb{D}}$. 

phism

The smooth Liouville vector field $w \mapsto \frac{-1}{2} w$ on $\mathbb{C}$ pulls back via the diffeomor-

$$
\tau \circ \psi:\left(\begin{array}{cc}
r e^{i \theta} & -\sqrt{1-r^{2}} \\
\sqrt{1-r^{2}} & r e^{-i \theta}
\end{array}\right) \in L_{0} \mapsto \sqrt{\frac{-1}{2} \ln \left(1-r^{2}\right)} e^{-i \theta} \in \mathbb{C},
$$

to the smooth Liouville vector field

$$
\begin{aligned}
X_{0}: u & \equiv\left(\begin{array}{cc}
r e^{i \theta} & -\sqrt{1-r^{2}} \\
\sqrt{1-r^{2}} & r e^{-i \theta}
\end{array}\right) \in L_{0} \\
& \mapsto\left(\begin{array}{cc}
\frac{\left(1-r^{2}\right) \ln \left(1-r^{2}\right)}{2 r} e^{i \theta} & \frac{\sqrt{1-r^{2}} \ln \left(1-r^{2}\right)}{2} \\
\frac{-\sqrt{1-r^{2}} \ln \left(1-r^{2}\right)}{2} & \frac{\left(1-r^{2}\right) \ln \left(1-r^{2}\right)}{2 r} e^{-i \theta}
\end{array}\right) \in T_{u} S U(2)
\end{aligned}
$$

on $L_{0}$, by differentiating the curve

$$
\begin{aligned}
& t \mapsto(\tau \circ \psi)^{-1}\left(e^{\frac{-1}{2} t} \sqrt{\frac{-1}{2} \ln \left(1-r^{2}\right)} e^{-i \theta}\right) \\
& =(\tau \circ \psi)^{-1}\left(\sqrt{\frac{-1}{2} e^{-t} \ln \left(1-r^{2}\right)} e^{-i \theta}\right) \\
& =(\tau \circ \psi)^{-1}\left(\sqrt{\frac{-1}{2} \ln \left[\left(1-r^{2}\right)^{e^{-t}}\right]} e^{-i \theta}\right) \\
& =\left(\begin{array}{cc}
\sqrt{1-\left(1-r^{2}\right)^{e^{-t}}} e^{i \theta} & -\sqrt{\left(1-r^{2}\right)^{e^{-t}}} \\
\sqrt{\left(1-r^{2}\right)^{e^{-t}}} & \sqrt{1-\left(1-r^{2}\right)^{e^{-t}}} e^{-i \theta}
\end{array}\right)
\end{aligned}
$$

at $t=0$.

Using the $U(1)$-action on $L_{0}$ that preserves the Poisson structure, we can get smooth scalings on other symplectic leaves of $S U(2)$. Actually since the above scaling $\phi_{t}$ on $u \in \overline{L_{0}}$ identified with $r e^{i \theta} \in \overline{\mathbb{D}}$ is along the radial direction, the scalings obtained in this way on all symplectic leaves can be described by one formula

$$
\begin{aligned}
\phi_{t}: u & =\left(\begin{array}{cc}
a & -\bar{c} \\
c & \bar{a}
\end{array}\right)=\left(\begin{array}{cc}
r e^{i \theta} & -s e^{-i \eta} \\
s e^{i \eta} & r e^{-i \theta}
\end{array}\right) \in S U(2) \mapsto \phi_{t}(u) \\
& =\left(\begin{array}{cc}
f_{t}(r) e^{i \theta} & -g_{t}(s) e^{-i \eta} \\
g_{t}(s) e^{i \eta} & f_{t}(r) e^{-i \theta}
\end{array}\right) \\
& =\left(\begin{array}{cc}
\sqrt{1-\left(1-r^{2}\right)^{t}} e^{i \theta} & -s^{t} e^{-i \eta} \\
s^{t} e^{i \eta} & \sqrt{1-\left(1-r^{2}\right)^{t}} e^{-i \theta}
\end{array}\right) \in S U(2)
\end{aligned}
$$

for $t>0$ with $s=\sqrt{1-r^{2}}$, where

$$
f_{t}(r)=\sqrt{1-\left(1-r^{2}\right)^{t}}
$$

and

$$
g_{t}(s)=s^{t}
$$


are continuous functions of $r, s \in[0,1]$ that vanish at $r, s=0$. By our construction, $\phi_{t}$ is smooth on each symplectic leaf of $S U(2)$, and it is clearly continuous globally on $S U(2)$. So we get a scaling $\phi_{t}, t>0$, of the standard Bruhat-Poisson structure of $S U(2)$.

Similarly, using the $U(1)$-action on $L_{0}$, we get the smooth Liouville vector field $X_{0}$ on $L_{0}$ extended to a (continuous) Liouville vector field

$$
\begin{aligned}
X: u & =\left(\begin{array}{cc}
r e^{i \theta} & -\sqrt{1-r^{2}} e^{-i \eta} \\
\sqrt{1-r^{2}} e^{i \eta} & r e^{-i \theta}
\end{array}\right) \in S U(2) \\
& \mapsto\left(\begin{array}{cc}
\frac{\left(1-r^{2}\right) \ln \left(1-r^{2}\right)}{2 r} e^{i \theta} & \frac{\sqrt{1-r^{2}} \ln \left(1-r^{2}\right)}{2} e^{-i \eta} \\
\frac{-\sqrt{1-r^{2}} \ln \left(1-r^{2}\right)}{2} e^{i \eta} & \frac{\left(1-r^{2}\right) \ln \left(1-r^{2}\right)}{2 r} e^{-i \theta}
\end{array}\right) \in T_{u} S U(2)
\end{aligned}
$$

on $(S U(2), \pi)$, which is smooth on $S U(2) \backslash U(1)$ and vanishes on $U(1)$, where we adopt the convention

$$
\left.\left(1-r^{2}\right)^{\beta}\left(\ln \left(1-r^{2}\right)\right)\right|_{r=1}:=\lim _{r \rightarrow 1-}\left(1-r^{2}\right)^{\beta}\left(\ln \left(1-r^{2}\right)\right)=0
$$

for any $\beta>0$.

2. $S U(n)$-homogeneous Poisson $\mathbb{S}^{2 n-1}$. In this section, we find explicitly a scaling of the $S U(n)$-covariant [LW2] or $S U(n)$-homogeneous [D2, VSo2] space $\mathbb{S}^{2 n-1}$ for all $n \geq 2$. We use $I_{n}$ to denote the $n \times n$ identity matrix.

By Soibelman's result [So], the symplectic leaves of the Bruhat-Poisson $S U(n)$ are exactly products of $t \in \mathbb{T}^{n-1} \subset S U(n)$ with the leaves $L_{0}$ in the $n-1$ canonically embedded basic $S U(2)$ 's arranged in various orders. More precisely, let $\iota_{k}: S U(2) \rightarrow$ $S U(n)$ be the canonical Poisson embedding defined by

$$
\iota_{k}(u):=I_{k-1} \oplus u \oplus I_{n-k-1}
$$

a block diagonal matrix for $u \in S U(2)$, and fix the reduced expression

$$
\sigma_{1} \sigma_{2} \sigma_{1} \sigma_{3} \sigma_{2} \sigma_{1} \ldots \sigma_{n-1} \sigma_{n-2} \ldots \sigma_{2} \sigma_{1}
$$

of the maximal element in the Weyl group $\mathcal{W}_{n}$ of $S U(n)$ with respect to the Bruhat ordering [H, Sh4], where $\left\{\sigma_{k}\right\}_{k=1}^{n}$ are the reflections associated with the fundamental roots determined by the embeddings $\left\{\iota_{k}\right\}_{k=1}^{n}$.

Soibelman's classification of symplectic leaves of Bruhat-Poisson compact simple Lie groups [So] (cf. the next section for more details) implies that there is a one-to-one correspondence

$$
(\delta, K) \leftrightarrow \delta L_{K}=\delta \iota_{K}\left(\left(L_{0}\right)^{m}\right)
$$

between symplectic leaves $\delta L_{K}$ of the Bruhat-Poisson $S U(n)$ and pairs $(\delta, K)$ of a point $\delta \in \mathbb{T}^{n-1} \subset S U(n)$ and an "admissible" sequence $K=\left(k_{1}, k_{2}, \ldots, k_{m}\right)$, i.e. a concatenation $J_{1} J_{2} \ldots J_{n}$ of sequences $J_{i}$ which are either empty or equal to $(i, i-$ $1, \ldots, i-k_{i}$ ) for some $0 \leq k_{i}<i$, where

$$
\iota_{K}:\left(u_{1}, \ldots, u_{m}\right) \in\left(L_{0}\right)^{m} \mapsto \iota_{k_{1}}\left(u_{1}\right) \ldots \iota_{k_{m}}\left(u_{m}\right) \in S U(n)
$$

and $L_{K}:=\iota_{K}\left(\left(L_{0}\right)^{m}\right)$ which is set to be $\left\{I_{n}\right\}$ if $K$ is an empty sequence. 
Note that the multiplication map

$$
\begin{aligned}
\iota & =\iota_{(n-1, \ldots, 1)}:\left(u_{1}, u_{2}, . ., u_{n-1}\right) \in S U(2)^{n-1} \\
& \mapsto \iota_{n-1}\left(u_{1}\right) \iota_{n-2}\left(u_{2}\right) \cdots \iota_{1}\left(u_{n-1}\right) \in S U(n),
\end{aligned}
$$

is Poisson on each product of symplectic leaves, and the map

$$
P_{n}: u \in S U(n) \rightarrow u\left(e_{1}\right) \in \mathbb{S}^{2 n-1}
$$

viewed as the $S U(n)$-action on $\mathbb{S}^{2 n-1}$ restricted to $S U(n) \times\left\{e_{1}\right\}$ is Poisson since $\left\{e_{1}\right\}$ is a 0-dimensional leaf of $\mathbb{S}^{2 n-1}$.

By induction, it is easy to verify that

$$
\begin{aligned}
& \left(P_{n} \circ \iota\right)\left(u_{1}, u_{2}, . ., u_{n-1}\right)=P_{n}\left(\iota_{n-1}\left(u_{1}\right) \iota_{n-2}\left(u_{2}\right) \cdots \iota_{1}\left(u_{n-1}\right)\right) \\
& =a_{n-1} e_{1}+\sum_{k=2}^{n-1}\left(a_{n-k} c_{n-k+1} \cdots c_{n-1}\right) e_{k}+\left(c_{1} c_{2} \cdots c_{n-1}\right) e_{n} \in \mathbb{S}^{2 n-1}
\end{aligned}
$$

for

$$
u_{k}=\left(\begin{array}{cc}
a_{k} & -c_{k} \\
c_{k} & \overline{a_{k}}
\end{array}\right)=\left(\begin{array}{cc}
r_{k} e^{i \theta_{k}} & -s_{k} e^{-i \eta_{k}} \\
s_{k} e^{i \eta_{k}} & r_{k} e^{-i \theta_{k}}
\end{array}\right) \in \delta_{\eta_{k}} L_{0} \subset S U(2)
$$

where

$$
\delta_{\eta}:=\operatorname{diag}\left(e^{-i \eta}, e^{i \eta}\right) \in U(1)=\mathbb{T} .
$$

By taking $a_{0}:=1$ and $a_{n-k} c_{n-k+1} \cdots c_{n-1}:=a_{n-1}$ when $k=1$, we can write more compactly

$$
P_{n} \circ \iota:\left(u_{1}, u_{2}, . ., u_{n-1}\right) \in S U(2)^{n-1} \mapsto z=\sum_{k=1}^{n}\left(a_{n-k} c_{n-k+1} \cdots c_{n-1}\right) e_{k} \in \mathbb{S}^{2 n-1}
$$

from which it is not hard to check that $P_{n} \circ \iota$ is surjective. Actually the following lemma provides some more specific details.

Lemma. The function $P_{n} \circ \iota$ restricted to

$$
L_{m, \eta_{1}, \ldots, \eta_{n-1}}:=\left\{\left(\delta_{\eta_{1}}, \ldots, \delta_{\eta_{n-1-m}}\right)\right\} \times\left(\delta_{\eta_{n-m}} L_{0}\right) \times \ldots \times\left(\delta_{\eta_{n-1}} L_{0}\right),
$$

with $\left(\delta_{\eta_{1}}, \ldots, \delta_{\eta_{n-1}}\right) \in \mathbb{T}^{n-1}$ and $1 \leq m \leq n-1$, is a (Poisson) diffeomorphism onto $\mathbb{S}_{\eta}^{2 m}$, where

$$
\eta:=-\eta_{n-1-m}+\eta_{n-m}+\cdots+\eta_{n-1}
$$

and

$$
\mathbb{S}_{\eta}^{2 m}:=\mathbb{S}^{2 n-1} \cap\left(\mathbb{C}^{m} \times e^{i \eta} \mathbb{R}_{>} \times\{0\}^{n-1-m}\right)
$$

In particular, $P_{n} \circ \iota$ maps $S U(2)^{n-1}$ onto $\mathbb{S}^{2 n-1}$ which is the disjoint union of $\mathbb{S}_{\eta}^{2 m}$ with $0 \leq m<n$ and $\eta \in[0,2 \pi)$.

Proof. Since $\iota\left(L_{m, \eta_{1}, \ldots, \eta_{n-1}}\right)$ is a symplectic leaf of $S U(n)$ and $P_{n}$ is a Poisson map, it suffices to show that $P_{n} \circ \iota$ restricted to $L_{m, \eta_{1}, \ldots, \eta_{n-1}}$ is a diffeomorphism onto $\mathbb{S}_{\eta}^{2 m}$ for $\eta:=-\eta_{n-1-m}+\eta_{n-m}+\cdots+\eta_{n-1}$. 
Note that the general condition $\left|a_{j}\right|^{2}+\left|c_{j}\right|^{2}=1$ for all $j$ implies for any $M<n$,

$$
\begin{aligned}
& \sum_{k=1}^{M}\left|a_{n-k}\left(c_{n-k+1} \cdots c_{n-1}\right)\right|^{2} \\
& =1-\left|c_{n-1}\right|^{2}+\sum_{k=2}^{M}\left(1-\left|c_{n-k}\right|^{2}\right)\left|c_{n-k+1}\right|^{2} \cdots\left|c_{n-1}\right|^{2} \\
& =1-\left|c_{n-1}\right|^{2}+\sum_{k=2}^{M}\left[\left|c_{n-k+1}\right|^{2} \cdots\left|c_{n-1}\right|^{2}-\left|c_{n-k}\right|^{2} \cdots\left|c_{n-1}\right|^{2}\right] \\
& =1-\left|c_{n-M}\right|^{2} \cdots\left|c_{n-1}\right|^{2} .
\end{aligned}
$$

For $z \in \mathbb{S}^{2 n-1} \subset \mathbb{C}^{n}$ and $\left(u_{1}, u_{2}, . ., u_{n-1}\right) \in S U(2)^{n-1}$,

$$
\left(u_{1}, u_{2}, . ., u_{n-1}\right) \in L_{m, \eta_{1}, \ldots, \eta_{n-1}} \cap\left(P_{n} \circ \iota\right)^{-1}(z)
$$

if and only if the conditions (i)

$$
c_{j}=e^{i \eta_{j}} \sqrt{1-\left|a_{j}\right|^{2}} \neq 0
$$

for all $j \geq n-m$, (ii) $a_{j}=e^{-i \eta_{j}}$ for all $j<n-m$, and (iii) for all $1 \leq k \leq n-1$,

$$
a_{n-k}\left(c_{n-k+1} \cdots c_{n-1}\right)=z_{k}
$$

(including $a_{n-1}=z_{1}$ when $k=1$ ) are satisfied.

Note that conditions (i)-(iii) imply that

$$
z_{m+1}=a_{n-m-1}\left(c_{n-m} \cdots c_{n-1}\right) \in e^{-\eta_{n-m-1}+\eta_{n-m}+\cdots+\eta_{n-1}} \mathbb{R}_{>}
$$

where $\mathbb{R}_{>}:=\{x \in \mathbb{R}: x>0\}$, and $z_{k}=0$ for all $k>m+1$ since $c_{n-m-1}=0$, or equivalently, $z \in \mathbb{S}_{\eta}^{2 m}$. So $P_{n} \circ \iota \operatorname{maps} L_{m, \eta_{1}, \ldots, \eta_{n-1}}$ into $\mathbb{S}_{\eta}^{2 m}$.

Also note that the condition (iii) implies

$$
1-\sum_{k=1}^{M}\left|z_{k}\right|^{2}=1-\sum_{k=1}^{M}\left|a_{n-k}\left(c_{n-k+1} \cdots c_{n-1}\right)\right|^{2}=\left|c_{n-M}\right|^{2} \cdots\left|c_{n-1}\right|^{2} .
$$

For $z \in \mathbb{S}_{\eta}^{2 m}$, we have $\left|z_{k}\right|<1$ for all $k \leq m$ and $z_{k}=0$ for all $k>m+1$, and $1-\sum_{k=1}^{M}\left|z_{k}\right|^{2}=0$ if and only if $M \geq m+1$. So under the condition (iii), we get $c_{n-k} \neq 0$ (and hence $u_{n-k} \in \mathbb{T} L_{0}$ ) for all $k<m+1$ and $c_{n-m-1}=0$ (and hence $\left.\left|a_{n-m-1}\right|=1\right)$, which then imply that for all $k \leq m+1$,

$$
a_{n-k}=z_{k}\left(c_{n-k+1} \cdots c_{n-1}\right)^{-1}
$$

is uniquely well-defined, and furthermore

$$
\left|a_{n-m-1}\right|=\left|z_{m+1}\left(c_{n-m} \cdots c_{n-1}\right)^{-1}\right|=\sqrt{\frac{\left|z_{m+1}\right|^{2}}{\left|c_{n-m}\right|^{2} \cdots\left|c_{n-1}\right|^{2}}}=\sqrt{\frac{\left|z_{m+1}\right|^{2}}{1-\sum_{k=1}^{m}\left|z_{k}\right|^{2}}}=1
$$

which combined with condition (ii) gives $a_{n-m-1}:=e^{-i \eta_{n-m-1}}$. For all $k>m+1$, we see that with $z_{k}=0$ and $c_{n-k+1} \cdots c_{n-1}=0, a_{n-k}=e^{-i \eta_{n-k}}$ is the unique 
solution for both conditions (ii) and (iii). Thus for any $z \in \mathbb{S}_{\eta}^{2 m}$, there is a unique $\left(u_{1}, u_{2}, . ., u_{n-1}\right) \in L_{m, \eta_{1}, \ldots, \eta_{n-1}}$ such that $\left(P_{n} \circ \iota\right)\left(u_{1}, u_{2}, . ., u_{n-1}\right)=z$.

So $P_{n} \circ \iota$ is a bijective smooth map from $L_{m, \eta_{1}, \ldots, \eta_{n-1}}$ to $\mathbb{S}_{\eta}^{2 m}$, whose inverse is also a smooth map $z \mapsto\left(u_{1}, u_{2}, . ., u_{n-1}\right)$ given by the formulas

$$
a_{n-k}:=\left\{\begin{array}{lll}
z_{k}\left(c_{n-k+1} \cdots c_{n-1}\right)^{-1}, & \text { if } \quad k \leq m+1 \\
e^{-i \eta_{n-k},} & \text { if } \quad k>m+1
\end{array}\right.
$$

and

for all $j$.

$$
c_{j}=e^{i \eta_{j}} \sqrt{1-\left|a_{j}\right|^{2}}
$$

THEOREM. There exists a (continuous and leafwise smooth) scaling $\psi_{t}, t>0$, of the standard Bruhat-Poisson structure of $\mathbb{S}^{2 n-1}$ for all $n \geq 2$.

Proof. Let $\phi_{t}$ be the scaling of $S U(2)$ obtained in the previous proposition. The scaling $\left(\phi_{t}\right)^{n-1}$ of the product Poisson manifold $S U(2)^{n-1}$ restricted to the symplectic leaf $L_{m, \eta_{1}, \ldots, \eta_{n-1}}$ induces, under the diffeomorphism $P_{n} \circ \iota$, a corresponding smooth scaling of $\mathbb{S}_{\eta}^{2 m}$ with $\eta:=-\eta_{n-1-m}+\eta_{n-m}+\cdots+\eta_{n-1}$, given by

$$
\begin{aligned}
& \left(\psi_{m, \eta_{1}, \ldots, \eta_{n-1}}\right)_{t}:\left(r_{n-1} e^{i \theta_{n-1}}\right) e_{1}+\sum_{k=2}^{m+1}\left(r_{n-k} s_{n-k+1} \cdots s_{n-1} e^{i\left(\theta_{n-k}+\eta_{n-k+1}+\cdots+\eta_{n-1}\right)}\right) e_{k} \\
& \mapsto\left[f_{t}\left(r_{n-1}\right) e^{i \theta_{n-1}}\right] e_{1}+\sum_{k=2}^{m+1}\left[f_{t}\left(r_{n-k}\right) g_{t}\left(s_{n-k+1}\right) \cdots g_{t}\left(s_{n-1}\right) e^{i\left(\theta_{n-k}+\eta_{n-k+1}+\cdots+\eta_{n-1}\right)}\right] e_{k}
\end{aligned}
$$

for $m \leq n-1$ with $r_{n-1-m}=1$ and $\theta_{n-1-m}=-\eta_{n-1-m}$, where $s_{j}=\sqrt{1-r_{j}^{2}}$ for all $j$, and as before, we take $r_{0}=a_{0}:=1$ when $m=n-1$.

Since the scaling $\left(\psi_{m, \eta_{1}, \ldots, \eta_{n-1}}\right)_{t}$ of $\mathbb{S}_{\eta}^{2 m}$ keeps the angle (argument) of each complex coefficient invariant, it depends only on $\eta$ and can be written as

$$
\begin{aligned}
& \left(\psi_{\eta}\right)_{t}: z=\left(r_{n-1} e^{i \beta_{1}}\right) e_{1}+\sum_{k=2}^{m+1}\left(r_{n-k} s_{n-k+1} \cdots s_{n-1} e^{i \beta_{k}}\right) e_{k} \in \mathbb{S}_{\eta}^{2 m} \mapsto \\
& \left(f_{t}\left(r_{n-1}\right) e^{i \beta_{1}}\right) e_{1}+\sum_{k=2}^{m+1}\left(f_{t}\left(r_{n-k}\right) g_{t}\left(s_{n-k+1}\right) \ldots g_{t}\left(s_{n-1}\right) e^{i \beta_{k}}\right) e_{k} \in \mathbb{S}_{\eta}^{2 m}
\end{aligned}
$$

with $r_{n-1-m}=1$ and $\theta_{n-1-m}=-\eta_{n-1-m}$.

Since $\mathbb{S}^{2 n-1}$ is a disjoint union of $\mathbb{S}_{n}^{2 m}$ with $0 \leq m<n$ and $\eta \in[0,2 \pi)$, we get a well-defined function $\psi_{t}: \mathbb{S}^{2 n-1} \rightarrow \mathbb{S}^{2 n-1}$ whose restriction to each symplectic leaf $\mathbb{S}_{\eta}^{2 m}$ is the smooth scaling $\left(\psi_{\eta}\right)_{t}$. Now it remains to show that $\psi_{t}$ is a homeomorphism of $\mathbb{S}^{2 n-1}$.

Note that if $\left|a_{n-j}\right|=1$ for some $j$, i.e. $u_{n-j} \in U(1)=\mathbb{T}$ is a diagonal $2 \times 2$ matrix, then

$$
\begin{aligned}
\left(P_{n} \circ \iota\right)\left(u_{1}, . ., u_{n-1}\right) & =\iota_{n-1}\left(u_{1}\right) \cdots \iota_{1}\left(u_{n-1}\right)\left(e_{1}\right) \\
& =\iota_{j}\left(u_{n-j}\right) \cdots \iota_{1}\left(u_{n-1}\right)\left(e_{1}\right) \in \mathbb{C}^{j} \times\{0\} \subset \mathbb{C}^{n} .
\end{aligned}
$$

So $\left(P_{n} \circ \iota\right)\left(u_{1}, \ldots, u_{n-1}\right) \in \mathbb{S}_{\eta}^{2 m}$ if and only if $\left|a_{n-m-1}\right|=1>\left|a_{k}\right|$ for all $k \geq n-m$, which implies that

$\left(^{*}\right) \quad\left(P_{n} \circ \iota\right)\left(u_{1}, \ldots, u_{n-1}\right)=\left(P_{n} \circ \iota\right)\left(I_{2}, \ldots, I_{2}, u_{n-m-1}, \ldots, u_{n-1}\right)$ 
with $u_{k} \in \mathbb{T} L_{0}$ for all $k \geq n-m$.

Now $S U(2)^{n-1}$ is a disjoint union of symplectic leaves $F=\prod_{k=1}^{n-1} A_{k}$ with $A_{k}$ either a singleton in $\mathbb{T} \subset S U(2)$ or a disk $e^{i \eta_{k}} L_{0} \subset S U(2)$ for some $\eta_{k}$. If $m$ is the largest index with $A_{n-m-1}$ a singleton, then $\prod_{k=1}^{n-1}\left\{I_{2}\right\} \times \prod_{k=n-m-1}^{n-1} A_{k}$ equals some $L_{m, \eta_{1}, \ldots, \eta_{n-1}}$ and by (*),

$$
\left(P_{n} \circ \iota\right)(F)=\left(P_{n} \circ \iota\right)\left(L_{m, \eta_{1}, \ldots, \eta_{n-1}}\right)=\mathbb{S}_{\eta}^{2 m} .
$$

It is not hard to see that the equality

$$
\psi_{t} \circ\left(P_{n} \circ \iota\right)=\left(P_{n} \circ \iota\right) \circ\left(\phi_{t}\right)^{n-1},
$$

clearly valid on $L_{m, \eta_{1}, \ldots, \eta_{n-1}}$, is also valid on $\left(P_{n} \circ \iota\right)(F)$ because of $\left(^{*}\right)$, for each symplectic leaf $F$ of $S U(2)^{n-1}$.

Thus we have the commuting diagram

$$
\begin{array}{ccc}
S U(2)^{n-1} & \left(\phi_{t}\right)^{n-1} & S U(2)^{n-1} \\
\downarrow P_{n} \circ \iota & \circlearrowright & \downarrow P_{n} \circ \iota \\
\mathbb{S}^{2 n-1} & \stackrel{\psi_{+}}{\rightarrow} & \mathbb{S}^{2 n-1}
\end{array}
$$

where $\mathbb{S}^{2 n-1}$ with its standard topology can be viewed as a quotient topological space of the compact Hausdorff space $S U(2)^{n-1}$ with $P_{n} \circ \iota$ as the quotient map, and $\psi_{t}$ can be viewed as a well-defined map on $\mathbb{S}^{2 n-1}$ induced by the continuous map $\left(\phi_{t}\right)^{n-1}$ on $S U(2)^{n-1}$. It is easy to see that the map $\psi_{t}$ on $\mathbb{S}^{2 n-1}$ is continuous.

So we have a well-defined continuous and leafwise smooth scaling $\psi_{t}, t>0$, on $\mathbb{S}^{2 n-1}$.

We remark that $\psi_{t}$ can be described by the formula

$$
\begin{aligned}
\psi_{t}: z= & {\left[r_{n-1} e^{i \beta_{1}}\right] e_{1}+\sum_{k=2}^{n-1}\left[r_{n-k} s_{n-k+1} \ldots s_{n-1} e^{i \beta_{k}}\right] e_{k}+\left[s_{1} s_{2} \ldots s_{n-1} e^{i \beta_{n}}\right] e_{n} \in \mathbb{S}^{2 n-1} \mapsto } \\
\psi_{t}(z)= & {\left[f_{t}\left(r_{n-1}\right) e^{i \beta_{1}}\right] e_{1}+\sum_{k=2}^{n-1}\left[f_{t}\left(r_{n-k}\right) g_{t}\left(s_{n-k+1}\right) \ldots g_{t}\left(s_{n-1}\right) e^{i \beta_{k}}\right] e_{k} } \\
& +\left[g_{t}\left(s_{1}\right) g_{t}\left(s_{2}\right) \ldots g_{t}\left(s_{n-1}\right) e^{i \beta_{n}}\right] e_{n} \in \mathbb{S}^{2 n-1}
\end{aligned}
$$

with $s_{j}=\sqrt{1-r_{j}^{2}}$ for all $j$.

3. Bruhat-Poisson compact simple Lie groups. In this section, we use the scaling $\phi_{t}$ of Bruhat-Poisson $S U(2)$ to construct a scaling for the standard BruhatPoisson compact simple Lie groups $K$ with a topology stronger than the standard one but still compatible with the original differential structure on each symplectic leaf.

For a simple complex Lie group $G$, we fix a root system $\Lambda$ with (positive) simple roots $\left\{\alpha_{i}\right\}_{i=1}^{r}$ for its Lie algebra $\mathfrak{g}$ and a corresponding Cartan-Weyl basis $\left\{X_{\alpha}\right\}_{\alpha \in \Lambda} \cup$ $\left\{H_{i}\right\}_{i=1}^{r}$ with $H_{i}=\left[X_{\alpha_{i}}, X_{-\alpha_{i}}\right]$ for each $i$. The real form (i.e. the +1 -eigenspace) for the antilinear involution $\omega: \mathfrak{g} \rightarrow \mathfrak{g}$ defined by $\omega\left(X_{\alpha}\right)=-X_{-\alpha}$ and $\omega\left(H_{i}\right)=-H_{i}$ for all $\alpha \in \Lambda$ and $1 \leq i \leq r$ is the Lie algebra $\mathfrak{g}$ of a maximal compact subgroup $K$ of $G$. We consider only $K$ endowed with the standard Bruhat-Poisson structure generated by the tensor

$$
\mathbf{r}=\frac{i}{2} \sum_{\alpha \in \Lambda_{+}}\left(X_{-\alpha} \otimes X_{\alpha}-X_{\alpha} \otimes X_{-\alpha}\right) \in \mathfrak{k} \wedge \mathfrak{k}
$$


There is a well-known canonical Poisson embedding $\iota_{i_{*}}: S U(2) \rightarrow K$ for each basic triple $\left\{X_{\alpha_{i}}, X_{-\alpha_{i}}, H_{i}\right\}, 1 \leq i \leq r$.

Recall that the Weyl group $W$ of $K$ is a Coxeter group [H] generated by $\left\{\sigma_{i}\right\}_{i=1}^{r}$ with $\left(\sigma_{i} \sigma_{j}\right)^{m_{i j}}=1$ for $m_{i i}=1$ and some $m_{i j} \in\{2,3,4,6\}$ if $i \neq j$, where $\sigma_{i}=\sigma_{\alpha_{i}}$ is the reflection on the dual $\mathfrak{h}^{*}$ of the Lie subalgebra $\mathfrak{h}:=\operatorname{Span}\left\{H_{i}\right\}_{i=1}^{r}$ determined by the root $\alpha_{i}$. If $w=\sigma_{i_{1}} \sigma_{i_{2}} \ldots \sigma_{i_{m}}$ is the shortest expansion of $w$ in $\sigma_{i}$ 's, then $\sigma_{i_{1}} \sigma_{i_{2}} \ldots \sigma_{i_{m}}$ is called a reduced expression for $w$ and $\ell(w):=m$ is the length of $w$. The Bruhat ordering on $W$ is the partial ordering generated by the relations $w_{1}<w_{2}$ satisfying $\sigma_{\alpha} w_{1}=w_{2}$ and $\ell\left(w_{1}\right)+1=\ell\left(w_{2}\right)$ for some simple root $\alpha$. It is known that there is a unique maximal element $\tilde{w}=\sigma_{l_{1}} \sigma_{l_{2}} \ldots \sigma_{l_{M}}$ in $W$ with respect to the Bruhat ordering $[\mathrm{H}]$ and every element of $W$ has a reduced expression embedded in the expression $\sigma_{l_{1}} \sigma_{l_{2}} \ldots \sigma_{l_{M}}[\mathrm{BB}]$ (i.e. obtainable by removing some $\sigma_{l_{j}}$ 's from $\tilde{w}$ ).

It is an interesting discovery [So] that the symplectic leaves $L$ of $K$ are in one-toone correspondence with elements $(\delta, w)$ of $\mathbb{T}^{r} \times W$ and hence with the irreducible $*_{-}$ representations $\pi_{L}$ of the algebra $C\left(K_{q}\right)^{\infty}$ of regular functions of a quantum group $K_{q}$. More explicitly, for each $(\delta, w) \in \mathbb{T}^{r} \times W$, we fix a reduced expression $\sigma_{i_{1}} \sigma_{i_{2}} \ldots \sigma_{i_{m}}$ for $w \in W$ such that $\sigma_{i_{1}} \sigma_{i_{2}} \ldots \sigma_{i_{m}}$ is embedded in $\sigma_{l_{1}} \sigma_{l_{2}} \ldots \sigma_{l_{M}}$, and then the set $\delta L_{w} \subset K$ is the corresponding symplectic leaf, where $L_{w}:=\iota_{w}\left(\left(L_{0}\right)^{m}\right)$ and

$$
\iota_{w}:\left(u_{1}, \ldots, u_{m}\right) \in\left(\overline{L_{0}}\right)^{m} \mapsto \iota_{i_{1}}\left(u_{1}\right) \ldots \iota_{i_{m}}\left(u_{m}\right) \in K .
$$

With $w=\sigma_{i_{1}} \sigma_{i_{2}} \ldots \sigma_{i_{m}}$ embedded in $\sigma_{l_{1}} \sigma_{l_{2}} \ldots \sigma_{l_{M}}$, we have $i_{k}=l_{j_{k}}$ for $k \leq m$ where

$$
1 \leq j_{1}<j_{2}<\ldots<j_{m} \leq M
$$

We define

$$
\mathcal{L}_{w}:=\mathbb{T}^{r} \times\left\{u \in\left(\overline{L_{0}}\right)^{M} \mid u_{j_{k}} \in L_{0} \text { for } k \leq \ell(w) \text { and } u_{j}=I_{2} \text { for other } j \text { 's }\right\} .
$$

Let $\mathcal{L} \subset \mathbb{T}^{r} \times\left(\overline{L_{0}}\right)^{M}$ be the union of these disjoint subsets $\mathcal{L}_{w}$ with $w \in W$. Then the continuous map

$$
\operatorname{id}_{\mathbb{T}^{r}} \times \iota_{\tilde{w}}:\left(\delta, u_{1}, \ldots, u_{M}\right) \in \mathbb{T}^{r} \times\left(\overline{L_{0}}\right)^{M} \mapsto \delta \iota_{l_{1}}\left(u_{1}\right) \ldots \iota_{l_{M}}\left(u_{M}\right) \in K
$$

sends $\mathcal{L}$ onto $K$. By viewing $K$ as a quotient space of $\mathcal{L}$, we get a quotient topology $\mathcal{T}$ on $K$ from $\mathcal{L}$ via the map $\left.\left(\operatorname{id}_{\mathbb{T}^{r}} \times \iota_{\tilde{w}}\right)\right|_{\mathcal{L}}$. By definition, $\mathcal{T}$ consists of sets $A \subset K$ with $\left(\operatorname{id}_{\mathbb{T}^{r}} \times \iota_{\tilde{w}}\right)^{-1}(A)$ open in $\mathcal{L}$, and is stronger than (i.e. contains) the original topology on $K$ and hence still Hausdorff. Furthermore, $\operatorname{id}_{\mathbb{T}^{r}} \times \iota_{\tilde{w}}$ is homeomorphic on each $\mathcal{L}_{w}$ and hence the topology $\mathcal{T}$ is compatible with the original differential structure on each symplectic leaf of $K$.

THEOREM. There exists a (continuous and leafwise smooth) scaling $\Phi_{t}, t>0$, of the standard Bruhat-Poisson structure of a compact simple Lie group $K$ when $K$ is endowed with the topology $\mathcal{T}$.

Proof. Since the Bruhat-Poisson structure on $K$ is multiplicative and $\mathbb{T}^{r}$ consists of 0 -dimensional leaves $\{\delta\}$ whose action by multiplication preserves the BruhatPoisson structure, the diffeomorphic map

$$
\iota_{\delta, w}:\left(u_{1}, u_{2}, . ., u_{m}\right) \in\left(L_{0}\right)^{m} \mapsto \delta \iota_{w}\left(u_{1}, u_{2}, . ., u_{m}\right) \in \delta L_{w}
$$

for $\delta \in \mathbb{T}^{r}$ and $w=\sigma_{i_{1}} \sigma_{i_{2}} \ldots \sigma_{i_{m}}$ is symplectic. So the smooth scaling

$$
\left(\phi_{t}\right)^{m}\left(u_{1}, u_{2}, . ., u_{m}\right)=\left(\phi_{t}\left(u_{1}\right), \phi_{t}\left(u_{2}\right), . ., \phi_{t}\left(u_{m}\right)\right)
$$


of the symplectic product space $\left(L_{0}\right)^{m}$ induces, via $\iota_{\delta, w}$, a smooth scaling $\left(\Phi_{\delta, w}\right)_{t}$ of the symplectic leaf $\delta L_{w}$.

Since $K$ is the disjoint union of its symplectic leaves $\delta L_{w}$ [W1], we get a family of well-defined functions $\Phi_{t}: K \rightarrow K$ which is the diffeomorphism $\left(\Phi_{\delta, w}\right)_{t}$ on each symplectic leaf $\delta L_{w}$. It remains to show that $\Phi_{t}$ is continuous on $K$ with respect to the topology $\mathcal{T}$.

By restricting to $\mathcal{L}$, we get a continuous map

$$
\left.\left(\mathrm{id}_{\mathbb{T}^{n-1}} \times\left(\phi_{t}\right)^{M}\right)\right|_{\mathcal{L}}: \mathcal{L} \subset \mathbb{T}^{r} \times\left(\overline{L_{0}}\right)^{M} \rightarrow \mathcal{L} \subset \mathbb{T}^{r} \times\left(\overline{L_{0}}\right)^{M}
$$

on $\mathcal{L}$. It is easy to see that $\left(\operatorname{id}_{\mathbb{T}^{r}} \times \iota_{\tilde{w}}\right)\left(\mathcal{L}_{w}\right)=\mathbb{T}^{r} L_{w}$ for all $w \in W$, and

$$
\left.\Phi_{t} \circ\left(\mathrm{id}_{\mathbb{T}^{r}} \times \iota_{\tilde{w}}\right)\right|_{\mathcal{L}}=\left.\left(\mathrm{id}_{\mathbb{T}^{r}} \times \iota_{\tilde{w}}\right)\right|_{\mathcal{L}} \circ\left(\operatorname{id}_{\mathbb{T}^{n-1}} \times\left.\left(\phi_{t}\right)^{M}\right|_{\mathcal{L}}\right)
$$

So when $K$ is endowed with the quotient topology $\mathcal{T}$ via $\left.\left(\operatorname{id}_{\mathbb{T}^{r}} \times \iota_{\tilde{w}}\right)\right|_{\mathcal{L}}$, the continuity of $\left.\left(\operatorname{id}_{\mathbb{T}^{n-1}} \times\left(\phi_{t}\right)^{M}\right)\right|_{\mathcal{L}}$ on $\mathcal{L}$ implies the continuity of $\Phi_{t}$ on $K$.

\section{REFERENCES}

[BB] A. Bjorner and F. Brenti, Combinatorics of Coxeter Groups, Springer, 2005.

[D1] V. G. Drinfeld, Quantum groups, Proc. I.C.M. Berkeley 1986, Vol. 1, pp. 789-820, Amer. Math. Soc., Providence, 1987.

[D2] On Poisson homogeneous spaces of Poisson Lie groups, Theo. Math. Phys., 95 (1993), pp. 226-227.

[H] H. Hiller, "Geometry of Coxeter Groups", Research Notes in Math. Vol. 54, Pitman, Boston, 1982.

[LW1] J. H. Lu And A. Weinstein, Poisson Lie groups, dressing transformations and Bruhat decompositions, J. Diff. Geom., 31 (1990), pp. 501-526.

[LW2] Classification of SU(2)-covariant Poisson structures on $\mathbb{S}^{2}$, Comm. Math. Phys., 135 (1991), pp. 229-231.

[N] G. NAGY, A deformation quantization procedure for $C^{*}$-algebras, J. Operator Theory, 44 (2000), pp. 369-411.

[Sh1] A. J. L. SHeu, Quantization of the Poisson SU(2) and its Poisson homogeneous space the 2-sphere, Comm. Math. Phys., 135 (1991), pp. 217-232.

[Sh2] Leaf-preserving quantizations of Poisson SU(2) are not coalgebra homomorphisms, Comm. Math. Phys., 172 (1995), pp. 287-292.

[Sh3] Symplectic leaves and deformation quantization, Proc. Amer. Math. Soc., 124 (1996), pp. 95-100.

[Sh4] Compact quantum groups and groupoid $C^{*}$-algebras, J. Func. Anal., 144 (1997), pp. 371-393.

[So] YA. S. SolBELman, The algebra of functions on a compact quantum group, and its representations, Algebra Analiz., 2 (1990), pp. 190-221. (Leningrad Math. J., 2 (1991), pp. 161-178.)

[VSo1] L. L. VaKSman And Ya. S. Soibelman, Algebra of functions on the quantum group SU(2), Func. Anal. Appl., 22 (1988), pp. 170-181.

[VSo2] The algebra of functions on the quantum group $S U(n+1)$, and odd-dimensional quantum spheres, Leningrad Math. J., 2 (1991), pp. 1023-1042.

[W1] A. Weinstein, The local structure of Poisson manifolds, J. Diff. Geom., 18 (1983), pp. 523557.

[W2] Poisson geometry, Diff. Geom. and its Appl., 9 (1998), pp. 213-238.

[W3] The modular automorphism group of a Poisson manifold, J. Geom. and Phys., 23 (1997), pp. 379-394. 